\title{
ON THE IRREDUCIBILITY OF AN INDUCED REPRESENTATION. II ${ }^{1}$
}

\author{
JOHN C. QUIGG
}

\begin{abstract}
A unitary representation induced from a normal subgroup of a separable locally compact group is irreducible if and only if the inducing representation is irreducible and the restriction of the induced representation to the normal subgroup is multiplicity-free.
\end{abstract}

1. Introduction. In the case where the quotient group is abelian, a result equivalent to that mentioned in the abstract (hereafter referred to as the Irreducibility Theorem) is proved in Quigg [11], which is based upon the author's thesis. This was accomplished in the abelian case using a duality result for induced representations which includes the separable case of Takesaki's duality theorem for crossed products of von Neumann algebras by abelian groups (Takesaki [12, Theorem 4.5]). Since then we have been able to remove the commutativity hypothesis using techniques motivated by the duality theory of crossed products (see, e.g., Enock [2], Nakagami and Takesaki [8], Strătilă, Voiculescu and Zsidó [11]). The motivation is sketched in Remark (i) at the end of this paper. However, the duality is now at the von Neumann algebraic level, as opposed to the representation theoretic level, with the consequence that we were unable to generalize the examples found during our work on the abelian case (Quigg [10, §5]).

As pointed out in Quigg [10, Introduction], the only new aspect of the Irreducibility Theorem is the necessity of the restriction condition. Its nonredundancy is proved in that paper by examples. It is important to notice that these examples can easily be rephrased in terms of group extensions, using the techniques of $\mathrm{N}$. Pedersen [9, §2]. For completeness, we outline here how this would be accomplished, preserving the notation of the examples. Let $\mathbf{T}$ denote the circle group (complex numbers of absolute value one), and define $K_{\mathrm{T}}=\mathbf{T} \times K$. Further define an action $\rho$ of $G$ on $K_{\mathrm{T}}$ by

$$
\rho_{\chi}(\lambda, n h)=(\langle h, \chi\rangle \lambda, n h),
$$

for $\chi \in G, \lambda \in \mathbf{T}, n \in N, h \in H$, and let $K_{\mathbf{T}} \times_{s} G$ be the associated semidirect product group. Now, the representation $\pi$ of $K$ can be extended to a representation $\pi_{\mathrm{T}}$ of $K_{\mathrm{T}}$ by

$$
\pi_{\mathbf{T}}(\lambda, k)=\lambda \pi(k)
$$

Received by the editors August 11, 1980 and, in revised form, November 15, 1981 and March 27, 1982. Presented at the 8th annual meeting of the American Mathematical Society, January 1981.

1980 Mathematics Subject Classification. Primary 22D30; Secondary 46L55. 
and it is clear that $\mathbb{Q}_{\pi_{\mathrm{T}}}=\mathbb{Q}_{\pi}$. Finally, it is easy to see that

$$
\mathbb{Q}_{\text {ind } \pi_{\mathrm{T}}} \simeq \mathbb{Q}_{\text {ind } \pi},
$$

where the induction on the left is in the Mackey sense, while that on the right is in the sense of twisted covariant systems.

The context for our induced representations is that of twisted covariant systems (which include separable locally compact group extensions), for which we fix our notation in the next section. A fuller discussion can be found in Quigg [10] or the original papers of Busby and Smith [1] and Leptin [3-6].

2. Preliminaries. A twisted covariant system $(G, A, \alpha, w)$ consists of a second countable locally compact group $G$, a separable Banach *-algebra $A$ having bounded approximate identity, a strongly Borel map $\alpha: G \rightarrow$ Aut $A$ (the isometric *-automorphisms of $A$ ), and a strictly Borel map $w: G \times G \rightarrow \mathcal{U}(M(A)$ ) (the unitary multipliers of $A$ ) satisfying:

$$
\begin{aligned}
\alpha_{e} & =\iota_{A}, \\
u \cdot(s . e) & =w(e, s)=1, \\
\alpha_{s} \alpha_{t} & =\alpha_{s t} \operatorname{Ad}_{w(s, t)} \\
w(r s . t) \alpha_{t}^{-1}(w \cdot(r . s)) & =w(r . s t) w(s, t) .
\end{aligned}
$$

A representation $(L, \pi)$ of $(G . A$. a. $w)$ in a separable Hilbert space $\mathcal{H}$ consists of a weakly Borel map $L: G \rightarrow \mathcal{U}\left(\bar{i}^{*}\right)$ (the unitary operators on $\mathcal{H}$ ) and a ${ }^{*}$-representation $\pi: A \rightarrow B(\tilde{H})$ (the bounded operators on $\tilde{K}^{-}$) satisfying:

$$
\begin{aligned}
& U(s) U(t)=U(s t) \pi \circ w(s, t), \\
& \operatorname{Ad}_{U(s)} \circ \pi=\pi \circ \alpha_{s} .
\end{aligned}
$$

The von Neumann algebra generated by $(U, \pi)$ is

$$
\mathbb{Q}_{(U, \pi)}=\mathbb{Q}_{U} \vee\left(\mathbb{Q}_{\pi}\right) \text {, }
$$

where $\mathcal{Q}_{U}$ and $\mathcal{Q}_{\pi}$ are the von Neumann algebras generated by $U$ and $\pi$, respectively.

For a representation $\pi$ of $A$ in $H$ the induced representation ind $\pi=\left(U_{\pi}, \tilde{\pi}\right)$ of $(G, A, \alpha, w)$ in $\tilde{\mathcal{K}}=L^{2}(G, \mathcal{H})=L^{2}(G) \otimes \mathcal{H}$ is defined by

$$
\begin{aligned}
& \left(U_{\pi}(s) \xi\right)(t)=\pi \circ w\left(s, s^{-1} t\right) \xi\left(s^{-1} t\right), \\
& (\tilde{\pi}(x) \xi)(t)=\pi \circ \alpha_{t}^{-1}(x) \xi(t) .
\end{aligned}
$$

A crucial result for our purposes, which is essentially Proposition 4.10 of Busby and Smith [1], is the following "saturation condition":

$$
\mathbb{Q}_{\text {ind } \pi} \vee\left(L^{\infty}(G) \otimes \mathbf{C}_{\mathcal{H}}\right)=\mathscr{B}\left(L^{2}(G)\right) \otimes \mathbb{Q}_{\pi} .
$$

3. Irreduciblity. Let $\pi$ be a representation of $A$ in $\mathcal{H}$.

THEOREM. The following are equivalent:

(i) ind $\pi$ is irreducible.

(ii) $\pi$ is irreducible and $\tilde{\pi}$ is multiplicity-free.

(iii) $\pi$ is irreducible with trivial stability subgroup and $\tilde{\pi}$ is type I. 
Proof. That (ii) and (iii) are equivalent follows from standard direct integral theory, and that (iii) implies (i) is well known, so we only show that (i) implies (ii).

First, the irreducibility of $\pi$ follows at once from (2.1).

For the other part, let $V$ be the unitary operator on $L^{2}(G) \otimes \tilde{\mathcal{C}}=L^{2}(G \times G, \mathcal{H})$ defined by

$$
(V \xi)(s, t)=\pi \circ w(s, t)^{*} \xi\left(t^{-1}, s t\right) .
$$

An easy calculation shows that $V$ intertwines ind $\tilde{\pi}$ with the representation $1_{L^{2}(G)} \otimes$ ind $\pi$, so that

$$
\begin{aligned}
\mathscr{B}\left(L^{2}(G)\right) \otimes \mathbb{Q}_{\tilde{\pi}} & =\left(L^{\infty}(G) \otimes \mathbf{C}_{\tilde{\mathcal{C}}}\right) \vee \mathbb{Q}_{\text {ind } \tilde{\pi}} \\
& =\left(L^{\infty}(G) \otimes \mathbf{C}_{\tilde{\mathcal{K}}}\right) \vee \operatorname{Ad}_{V}\left(\mathbf{C}_{L^{2}(G)} \otimes \mathbb{Q}_{\text {ind } \pi}\right) \\
& =\operatorname{Ad}_{V}\left(\operatorname{Ad}_{V^{*}}\left(L^{\infty}(G) \otimes \mathbf{C}_{\tilde{\mathscr{C}}}\right) \vee\left(\mathbf{C}_{L^{2}(G)} \otimes \Re(\tilde{\mathcal{K}})\right)\right) .
\end{aligned}
$$

Now, for $f$ in $L^{\infty}(G)$, it is readily verified that

$$
\operatorname{Ad}_{V^{*}}\left(f \otimes 1_{\tilde{\mathcal{C}}}\right)=\rho^{*}\left(f \otimes 1_{L^{2}(G)}\right) \otimes 1_{\mathcal{K}},
$$

where $\rho^{*}$ is the automorphism of $L^{\infty}(G) \otimes L^{\infty}(G)$ induced by the homeomorphism $\rho:(s, t) \rightarrow(t s, t)$ of $G \times G$. Moreover, $\rho^{*}$ acts trivially on $\mathrm{C}_{L^{2}(G)} \otimes L^{\infty}(G)$, so that

$$
\begin{aligned}
\rho^{*}\left(L^{\infty}(G)\right. & \left.\otimes \mathbf{C}_{L^{2}(G)}\right) \vee\left(\mathbf{C}_{L^{2}(G)} \otimes L^{\infty}(G)\right) \\
& =\rho^{*}\left(L^{\infty}(G) \otimes \mathbf{C}_{L^{2}(G)}\right) \vee \rho^{*}\left(\mathbf{C}_{L^{2}(G)} \otimes L^{\infty}(G)\right) \\
& =\rho^{*}\left(L^{\infty}(G) \otimes L^{\infty}(G)\right) \\
& =L^{\infty}(G) \otimes L^{\infty}(G) .
\end{aligned}
$$

Hence, since

$$
\mathbf{C}_{L^{2}(G)} \otimes \mathscr{B}(\tilde{\mathcal{H}})=\left(\mathbf{C}_{L^{2}(G)} \otimes L^{\infty}(G) \otimes \mathbf{C}_{\mathscr{C}}\right) \vee\left(\mathbf{C}_{L^{2}(G)} \otimes \mathscr{B}(\tilde{\mathcal{H}})\right),
$$

we have

$$
\begin{aligned}
& \operatorname{Ad}_{V^{*}}\left(L^{\infty}(G) \otimes \mathbf{C}_{\tilde{K}}\right) \vee\left(\mathbf{C}_{L^{2}(\tilde{G})} \otimes \mathscr{B}(\tilde{\mathcal{H}})\right) \\
& =\left(\rho^{*}\left(L^{\infty}(G) \otimes \mathbf{C}_{L^{2}(G)}\right) \otimes \mathbf{C}_{\mathcal{X}}\right) \vee\left(\mathbf{C}_{L^{2}(G)} \otimes L^{\infty}(G) \otimes \mathbf{C}_{\mathcal{X}}\right) \\
& \vee\left(\mathbf{C}_{L^{2}(G)} \otimes \mathscr{B}(\tilde{\mathcal{C}})\right) \\
& =\left(L^{\infty}(G) \otimes L^{\infty}(G) \otimes \mathbf{C}_{\mathcal{C}}\right) \vee\left(\mathbf{C}_{L^{2}(G)} \otimes \Re(\tilde{\mathcal{K}})\right) \\
& =\left(L^{\infty}(G) \otimes \mathbf{C}_{\tilde{\mathcal{K}}}\right) \vee\left(\mathbf{C}_{L^{2}(G)} \otimes L^{\infty}(G) \otimes \mathbf{C}_{\mathcal{K}}\right) \\
& \vee\left(\mathbf{C}_{L^{2}(G)} \otimes \mathscr{B}(\tilde{\mathcal{H}})\right) \\
& =\left(L^{\infty}(G) \otimes \mathbf{C}_{\tilde{\mathscr{C}}}\right) \vee\left(\mathbf{C}_{L^{2}(G)} \otimes \mathscr{B}(\tilde{\mathcal{F}})\right) \\
& =L^{\infty}(G) \otimes \mathscr{B}(\tilde{\mathcal{H}}) \text {. }
\end{aligned}
$$

Thus $\Re\left(L^{2}(G)\right) \otimes \mathbb{Q}_{\tilde{\pi}}$ is spatially isomorphic to $L^{\infty}(G) \otimes \mathscr{F}(\tilde{\mathcal{H}})$. Taking commutants, one sees that $\mathbb{Q}_{\tilde{\pi}}^{\prime}$ is isomorphic to $L^{\infty}(G)^{\prime}=L^{\infty}(G)$, which makes $\tilde{\pi}$ multiplicity free. Q.E.D. 
REMARKS. (i) The motivation for the above proof involves crossed products of von Neumann algebras by coactions of groups (perhaps "cocrossed products" would be a more appropriate term). Using techniques which are now familiar, we have obtained the following "duality theorem": there is a coaction of $G$ on $\mathbb{Q}_{\text {ind } \pi}$ producing a crossed product isomorphic to $\mathscr{B}\left(L^{2}(G)\right) \otimes \mathbb{Q}_{\tilde{\pi}}$. Also, it is a special case of a perturbation result of Enock [2, Proposition II.3] that the crossed product of $\mathscr{B}(\mathcal{K})$ (here $\mathscr{K}$ is an arbitrary Hilbert space) by a unitarily implemented coaction of $G$ is isomorphic to $L^{\infty}(G) \otimes \mathscr{B}(\mathscr{K})$. In fact, the same result characterizes "inner crossed products" by coactions. The product of the unitary operators which implement the above isomorphisms is the operator $V$ occurring in the above proof.

(ii) Corollaries 4.4-4.6 in Quigg [10] follow in the present generality with the same proofs.

ACKNOWLEDGEMENTS. The author is indebted to the referee for (1) greatly simplifying the proof of the theorem, which had previously taken the form outlined in Remark (i), and (2) pointing out the need for the discussion in the second paragraph of the Introduction. The author would again like to express his gratitude to Robert C. Busby for suggesting this problem to him.

\section{REFERENCES}

1. R. Busby and H. Smith, Representations of twisted group algebras, Trans. Amer. Math. Soc. 149 (1970), 503-537.

2. M. Enock, Produit croisé d'une algèbre de von Neumann par une algèbre de Kac, J. Funct. Anal. 26 (1977), 16-47.

3. H. Leptin, Verallgemeinerte $L^{1}$-Algebren, Math. Anal. 159 (1965), 51-76.

4. Verallgemeinerte $L^{1}$-Algebren und projektive Darstellungen lokal kompakter Gruppen. I. Invent. Math. 3 (1967), 257-281.

5. , Verallgemeinerte $L^{1}$-Algebren und projektive Darstellungen lokal kompakter Gruppen. II, Invent. Math. 4 (1967), 68-86.

6. ___ Darstellungen verallgemeinerter $L^{1}$-Algebren, Invent. Math. 5 (1968), 192-215.

7. _ Darstellungen verallgemeinerter $L^{1}$-Algebren. II, Lecture Notes in Math., vol. 247, SpringerVerlag, Berlin and New York, 1972, pp. 251-307.

8. Y. Nakagami, and M. Takesaki, Duality for crossed products of von Neumann algebras, Lecture Notes in Math., vol. 731, Springer-Verlag, Berlin and New York, 1979.

9. N. Pedersen, Duality for induced representations and induced weights, Univ. of Copenhagen, preprint, No. 7, 1980.

10. J. Quigg, On the irreducibility of an induced representation, Pacific J. Math. 93 (1981), 163-179.

11. S. Strătilă, D. Voiculescu and L. Zsidó, On crossed products. I, Rev. Roumaine Math. Pures Appl. 21 (1976), 1411-1449.

12. M. Takesaki, Duality for crossed products and the structure of von Neumann algebras of type III, Acta. Math. 131 (1973), 249-310.

Department of Mathematics, Arizona State University, Tempe, Arizona 85281 